\title{
Vortex mass and viscosity in Josephson-junction arrays
}

\author{
Ulrich Eckern \\ Kernforschungszentrum Karlsruhe, Institut für Nukleare Festkörperphysik, Postfach 3640, W-7500 Karlsruhe 1, \\ Federal Republic of Germany \\ Edouard B. Sonin \\ A. F. Ioffe Physico-Technical Institute, Russian Academy of Sciences, 194021 St. Petersburg, Russia
}

(Received 14 July 1992)

\begin{abstract}
We study, on the basis of the continuum limit of the standard model for Josephson-junction arrays, the motion of single vortices by directly deriving the equation of motion for the vortex coordinate. Thereby we confirm and extend recent studies by U. Geigenmüller et al. [this issue, Phys. Rev. B 47, 348 (1992)]. In addition, we discuss the case of a triangular lattice, where due to a lower potential barrier and a higher vortex mass, ballistic motion is more likely to be observable.
\end{abstract}

\section{INTRODUCTION}

The possibility of ballistic vortex motion in underdamped Josephson-junction arrays (JJA's), based on the notion that a mass proportional to the nearest-neighbor capacitance $C$ can be assigned to a vortex, has recently attracted considerable attention, experimentally ${ }^{1,2}$ as well as theoretically. ${ }^{3-8}$ In particular, numerical ${ }^{7,8}$ and analytical $^{8}$ arguments indicate that ballistic motion is only possible for very small velocities, $v<0.1 \omega_{p}$; here $\omega_{p}$ denotes the single junction plasma frequency, $\left(\hbar \omega_{p}\right)^{2}=8 E_{J} E_{C}$, where $E_{J}=(\hbar / 2 e) I_{J}$ is the Josephson coupling energy, and $E_{C}=e^{2} / 2 C$ (the nearest-neighbor distance is chosen to be unity). Furthermore, for $v$ above this value, the vortex motion is strongly affected by the coupling to the plasma oscillations, ${ }^{7,8}$ which results in a strong viscous force even in the absence of ohmic losses.

As pointed out in Ref. 8, the essential features of this effect can be understood within the continuum limit of the standard JJA model. The analysis of that paper, in addition to numerical simulations, has been based on an energy balance argument: The rate of change of the plasma mode (spin-wave) energy is equated to the energy transferred to the vortex by the external force per unit time, from which the velocity-force relation can be computed. In this work, we confirm and extend the analysis of Ref. 8 by directly deriving the equation of motion for the vortex coordinate, following the procedure discussed in Refs. 3-5. In addition, we present explicitly the analogous results for a triangular JJA (there the pinning potential due to the lattice structure is reduced by about four, ${ }^{9}$ and the mass is enhanced by about two, ${ }^{10}$ compared to the square lattice), where ballistic motion is more likely to be observable, and presumably has been observed. ${ }^{2}$

\section{VORTEX DYNAMICS}

We follow closely, in spirit and notation, the approach discussed in detail in Ref. 4, which starts from the (Euclidean) action for the order-parameter phases on each superconducting island. In addition to the above- mentioned parameters, we define $M=\hbar^{2} C / 4 e^{2}$, $m=\hbar^{2} C_{0} / 4 e^{2} \quad\left(C_{0}\right.$ is the ground capacitance; $\left.\omega_{p}^{2}=E_{J} / M\right)$, and $\mathbf{f}=(\hbar / 2 e) \mathbf{I}_{x}$, where $\mathbf{I}_{x}$ is the external current. Furthermore, ohmic losses (due to parallel shunts) are characterized by a function $B\left(\tau-\tau^{\prime}\right)$, defined through its Fourier transform $B(\omega)=-2 \eta /|\omega|$, $\eta=(\hbar / 2 \pi) R_{0} / R, R_{0}=\pi \hbar / 2 e^{2}$, where $R$ denotes the shunt resistor. The effective action in the continuum limit is conveniently written as follows:

$$
S=S_{0}+S_{1}+S_{f}+S_{D}
$$

where

$$
\begin{aligned}
& S_{0}=\frac{1}{2} E_{J} \int d^{2} r \int d \tau(\nabla \phi)^{2}, \\
& S_{1}=\frac{1}{2} \int d^{2} r \int d \tau\left[m \dot{\phi}^{2}+M(\nabla \dot{\phi})^{2}\right], \\
& S_{f}=-\int d^{2} r \int d \tau \mathbf{f} \cdot \nabla \phi, \\
& S_{D}=-\frac{1}{4} \int d^{2} r \int d \tau d \tau^{\prime} B\left(\tau-\tau^{\prime}\right)(\nabla \dot{\phi})_{\tau^{\prime}}(\nabla \dot{\phi})_{\tau^{\prime}} .
\end{aligned}
$$

Note that there are subtle questions related to the order of derivatives (compare Ref. 5) for a vortex configuration. In the next step, we consider the equation of motion for $\phi(r, \tau)$ and separate explicitly the vortex from the spinwave part: $\phi=\phi^{V}+\phi^{S}, \nabla^{2} \phi^{V}=0$. Then, solving for the spin-wave part and inserting the result into (1), we arrive at an effective action in terms of the vortex coordinates 4 $\left\{\mathbf{r}_{j}(\tau)\right\}$. For convenience, the result is presented in the form $S[\phi]=S_{0}\left[\phi^{V}\right]+S_{f}[\phi]+S_{2}$, thereby defining $S_{2}$. Clearly, $S_{0}\left[\phi^{V}\right]$ represents the vortex-vortex interaction, logarithmic at large distances, and $S_{f}$ the Lorentz force contribution. ${ }^{4}$ In an intermediate step, $S_{2}$ is given by

$S_{2}=\frac{1}{2} \int \frac{d^{2} q}{(2 \pi)^{2}} \int d \tau d \tau^{\prime}\left(\nabla \dot{\phi}^{V}\right)_{-\mathbf{q}, \tau} g\left(\tau-\tau^{\prime}, q\right)\left(\nabla \dot{\phi}^{V}\right)_{\mathbf{q}, \tau^{\prime}}$

where 


$$
g(\omega, q)=\frac{E_{J}}{|\omega|} \frac{m|\omega|+q^{2}(M|\omega|+\eta)}{m \omega^{2}+q^{2}\left(M \omega^{2}+\eta|\omega|+E_{J}\right)},
$$

and, finally,

$$
S_{2}\left[\left\{\mathbf{r}_{j}\right\}\right]=\frac{1}{2} \sum_{i, j} e_{i} e_{j} \int d \tau d \tau^{\prime} \dot{r}_{i}^{\alpha}(\tau) \dot{r}_{j}^{\beta}\left(\tau^{\prime}\right) G_{\alpha \beta}\left(\tau-\tau^{\prime}, \Delta \mathbf{r}_{i j}\right),
$$

with $\Delta \mathbf{r}_{i j}=\mathbf{r}_{i}(\tau)-\mathbf{r}_{j}\left(\tau^{\prime}\right) ; e_{j}= \pm 1$ denotes the vorticity of the vortex with coordinate $\mathbf{r}_{j}$. In addition,

$$
G_{\alpha \beta}(\tau, \mathbf{r})=\int \frac{d^{2} q}{q^{2}}(\hat{z} \times \mathbf{q})_{\alpha}(\hat{z} \times \mathbf{q})_{\beta} g(\tau, q) e^{i \mathbf{q} \cdot \mathbf{r}},
$$

where $\hat{z}=(0,0,1)$. Note that a suitable cutoff is required for the $q$ integration, which we choose of the form $\exp \left(-q / q_{c}\right)$, with $q_{c}^{2}=2 \pi$ for the square lattice.

From Eq. (8) it is straightforward to derive the classical equations of motion for the vortex coordinates $\left\{\mathbf{r}_{j}(t)\right\}$, following standard analytical continuation procedures (see, e.g., Ref. 11). For simplicity, we concentrate in the following on the motion of a single vortex (positive vorticity), whose coordinate is denoted by $r(t)$. The result is

$\int_{-\infty}^{+\infty} d t^{\prime}\left\{\dot{G}_{\gamma \beta}^{R}\left(t-t^{\prime}, \mathbf{r}(t)-\mathbf{r}\left(t^{\prime}\right)\right) \dot{r}^{\beta}\left(t^{\prime}\right)+\dot{r}^{\alpha}(t) \dot{r}^{\beta}\left(t^{\prime}\right)\left[\nabla_{\alpha} G_{\gamma \beta}^{R}\left(t-t^{\prime}, \mathbf{r}(t)-\mathbf{r}\left(t^{\prime}\right)\right)-\nabla_{\gamma} G_{\alpha \beta}^{R}\left(t-t^{\prime}, \mathbf{r}(t)-\mathbf{r}\left(t^{\prime}\right)\right)\right]\right\}=\mathcal{F}_{x}^{\gamma}$,

where $\mathfrak{F}_{x}=-2 \pi(\hat{z} \times \mathbf{f})$; summation over repeated indices is implied. $G^{R}$ is given by an expression similar to (9), with $g(\tau, q) \rightarrow g^{R}(t, q)$, where $g^{R}(\omega, q)=g(|\omega| \rightarrow-i \omega$ $+0, q)$ is the retarded continuation of $g$, and $\dot{G}^{R}$ is obtained analogously with $-i \omega g^{R}$ replacing $g^{R}$.

In order to establish the connection with previous results $^{4,5}$ (compare also Ref. 12 where the vortex mass was introduced first), consider $m=0$, and small frequencies and velocities such that we need to retain only the contribution in (10) linear in the coordinate. Using $\dot{g}^{R}=-i \omega M+\eta$ appropriate for this limit, we find

$$
2 \pi^{2}(M \ddot{\mathbf{r}}+\eta \dot{\mathbf{r}})=\mathscr{F}_{x}
$$

from which we identify the "adiabatic" mass and viscosity of the vortex, $M=2 \pi^{2} M$ and $2 \pi^{2} \eta$, respectively. In particular, the value for the mass, which is obtained here by a simple argument [compare Eq. (16)], has been confirmed by various numerical investigations. 4,13

\section{BEYOND THE ADIABATIC LIMIT}

Clearly, it is difficult to solve (10) for a time-dependent external force beyond the linear regime. However, for a constant force we expect the vortex to move at constant velocity, and we study this case in the following. For a constant velocity, we find that only the first term on the left-hand side of (10) survives (the other two terms cancel) and, in addition, we confirm that the "viscous" force is in the same direction as the velocity (i.e., there is no indication of a Magnus force). Thus, putting $\mathbf{v}=(v, 0)$ and $\mathfrak{F}_{x}=\left(F_{x}, 0\right)$ for simplicity, we obtain

$$
\mathcal{L}(v) \equiv v \int d^{2} q \frac{q_{y}^{2}}{q^{2}} e^{-q / q_{c}} \dot{g}^{R}\left(q_{x} v, q\right)=\mathscr{F}_{x},
$$

where the cutoff is included for completeness. Specializing further to the limit of vanishing ohmic losses, $\eta \rightarrow 0$, we note that

$$
\operatorname{Re} \dot{g}^{R}(\omega, q)=\frac{\pi}{2} E_{J}\left\{\delta\left(\omega-\omega_{q}\right)+\delta\left(\omega+\omega_{q}\right)\right\},
$$

where $\omega_{q}$ is the spin-wave dispersion; $\omega_{q}^{2}=c^{2} q^{2} /$ $\left(1+\lambda^{2} q^{2}\right)$, where the spin-wave velocity $c$ and the screening length $\lambda$ are given by $c^{2}=E_{J} / m$ and $\lambda^{2}=M / m$, respectively. Clearly, (12) describes the dissipation in the vortex motion due to the creation of spin-wave excitations. Using (12) and (13), it becomes apparent that these results coincide exactly with the expression obtained in Ref. 8, for the case of an optical spectrum $\left(m=0 \rightarrow \omega_{q}=\omega_{p}\right)$ studied there. In particular, the viscous force is characterized by a sharp onset of dissipation for a velocity above $\simeq 0.1 \omega_{p}$, which is apparent from the small velocity behavior of $\mathcal{L}^{(}(v)$ :

$$
M \gg m, v \rightarrow 0: \quad \mathcal{L}(v) \simeq 2 \pi E_{J}\left(\frac{\pi v}{2 \omega_{p}}\right)^{1 / 2} q_{c}^{3 / 2} \exp \left(-\frac{\omega_{p}}{v q_{c}}\right) .
$$

A reasonable criterion to determine a critical velocity, $v_{c}$, appears to be (compare Ref. 8, Fig. 5) the condition $\mathcal{L}\left(v_{c}\right)=0.03 \times 2 \pi E_{J}$, which is, in fact, chosen such that (14) leads to $v_{c} \simeq 0.1 \omega_{p}$. (This procedure is used mainly for comparison with the triangular JJA, see below.) Note that the viscous force is bounded from above, $\mathcal{L}(v \rightarrow \infty)=2 \pi E_{J} q_{c}$; however, the regime of very high velocities is physically not relevant since it corresponds to a current exceeding the critical current of a single junction.
The dissipation discussed here (and in Ref. 8) is, in fact, Cherenkov radiation of spin waves. The threshold for this process is determined by the maximal wave vector on the border of the Brillouin zone; and introducing a smooth cutoff in (12) actually assumes that Umklapp processes are possible. Clearly, the dissipation at small velocities, subcritical or of the order of the critical, is determined by the regime of large wave vectors; thus, the continuum limit theory cannot be expected to give (numerically) precise results. 
In addition, for small velocities, the vortex velocity is not time independent but rather strongly affected by the (periodic) pinning potential due to the lattice, neglected in the continuum approximation. Using the well-known estimate of the potential barrier ${ }^{9,4}$ and the adiabatic mass $M \simeq 2 \pi^{2} M$, and ignoring the dissipation for a moment, the vortex energy is given by

$$
E=\frac{1}{2} M \dot{x}^{2}+\kappa E_{J}[1-\cos (2 \pi x)]
$$

with $\kappa \simeq 0.1$. From this expression, considering energies slightly above the potential maximum, $2 \kappa E_{J}$, it follows that the maximal velocity is given by $\sim 0.14 \omega_{p}$, already above the critical velocity $v_{c}$. Thus, even given the uncertainties in the numerical coefficients, it appears that there is hardly any window for classical nondissipative motion for the square lattice JJA.

We remark that a physically similar mechanismshowing a sharp onset of dissipation due to the creation of a local excitation - was studied earlier (compare Ref. 4 , Sec. V), where the damping of vortex motion due to the creation of quasiparticle-pair excitations was considered: There, of course, the critical velocity is determined by the superconducting energy gap, $\Delta$, instead of the plasma frequency as presently. Here we neglect quasiparticle excitations, thus assuming $\hbar \omega_{p} \ll \Delta$.

\section{THE TRIANGULAR LATTICE}

In this section, we present explicitly the corresponding results for the triangular JJA, which can be obtained from the above by simple scaling. Considering the expression (1) for the effective action, we note (i) that the gradient terms in (2), (3), and (5) have to be multiplied by $\frac{3}{2}$, reflecting that, for a triangular lattice, we have six nearest neighbors instead of four for the square lattice; and (ii) there is an overall factor $2 / \sqrt{3}$ in all terms, reflecting that the unit cell area presently is $\sqrt{3} / 2$ instead of unity. As a result, we thus have the following replacements: $\quad m \rightarrow 2 m / \sqrt{3}, \quad\left(E_{J}, M, \eta\right) \rightarrow \sqrt{3}\left(E_{J}, M, \eta\right)$, $\mathbf{f} \rightarrow 2 \mathbf{f} / \sqrt{3}$. In addition, the first Brillouin zone area is given by $(2 \pi)^{2} \times 2 / \sqrt{3}$; hence, $q_{c \Delta}^{2}=4 \pi / \sqrt{3}$ is the appropriate cutoff. Of course, the plasma frequency, $\omega_{p}$, is not modified, while the spin-wave velocity is now given by $c_{\Delta}^{2}=\frac{3}{2} E_{J} / m$.

Consider then, as above, the adiabatic mass of the vortex, which follows, to be given by ${ }^{10}$

$$
M_{\Delta}=\frac{\sqrt{3}}{2} M \int d^{2} q e^{-q / q_{c \Delta}}=4 \pi^{2} M
$$

and thus is by a factor 2 larger than the square lattice value. Furthermore, the velocity-force relation is given by (12)-(14), with the above replacements. Defining again the classical critical velocity by the condition $\mathcal{L}_{\Delta}\left(v_{c \Delta}\right)=0.03 \times 2 \pi E_{J}$, we obtain $v_{c \Delta} \simeq 0.08 \omega_{p}$, slightly smaller than for the square lattice. Thus, while the critical velocity is not much different, it is more important that the potential barrier is reduced ${ }^{9,10}$ by about a factor 4. Hence, considering the vortex energy $E \sim 4 \kappa_{\Delta} E_{J} \sim 0.1 E_{J}$ as an example, the velocity will be in the range $(0.05-0.07) \omega_{p}$, just below $v_{c \Delta}$. Thus, roughly, classical nondissipative vortex motion for the triangular lattice is possible for energies between the barrier height and twice its value.

\section{DISCUSSION}

Based on the continuum limit of the standard JJA model, we have confirmed and extended recent studies of vortex motion. ${ }^{7,8}$ In particular, it is found that classical nondissipative motion (even in the absence of ohmic losses) is only possible for very small velocities $v<v_{c}$, while for $v>v_{c}$, the creation of spin-wave excitations leads to a constant velocity in the presence of a constant force. In the present paper, we directly derived the equation of motion for the vortex coordinate, and presented an expression for the velocity-force relation, valid for finite $m$ and $\eta$ and thus more general than the result given in Ref. 8 but, of course, in agreement with the result for the special case considered there $(m=0, \eta=0)$. We emphasize again that the results obtained from the continuum model are quantitatively questionable to some extent, since they rely on certain assumptions concerning the short-distance behavior; clearly, however, they are most helpful in analyzing and supplementing numerical investigations. $^{7,8}$

For completeness, we briefly discuss the limit $M<m$ $\left(c<<\omega_{p}\right)$, in which case the spin-wave spectrum essentially is linear, $\omega_{q} \simeq c q$. In particular, in the extreme limit $M=0$, it is clear that $\mathcal{L}(v<c)=0$, while strong dissipation sets in when the vortex velocity becomes equal to the spin-wave velocity. Using (12) and (13), and putting $\beta=v / c$ for brevity, we find

$$
M=0, v>c: \quad \mathcal{L}(v)=2 \pi E_{J} q_{c}\left[\beta^{2}-1\right]^{1 / 2} / \beta .
$$

This result, for small but finite $M$, actually applies for $v$ not too close to $c,\left(\beta^{2}-1\right)^{1 / 2}>v / \omega_{p}$. For $v \rightarrow c$, and $v<c$, the deviation of $\omega_{q}$ from linearity has to be taken into account. For example, we find

$$
M \ll m, v=c: \mathcal{L}(c)=2 \pi E_{J} q_{c} c q_{c} / \omega_{p} .
$$

Furthermore, for $v$ less than but not too close to $c$, the dissipative force is given by

$$
v<c: \quad \mathcal{L}(v) \simeq 2 \pi E_{J}\left[\frac{\pi v}{2 \omega_{p}}\right]^{1 / 2} q_{c}^{3 / 2}\left(1-\beta^{2}\right)^{1 / 4} \exp \left[-\frac{\omega_{p}}{v q_{c}}\left(1-\beta^{2}\right)^{1 / 2}\right]
$$

which connects with expression (18) for $\left(1-\beta^{2}\right)^{1 / 2} \sim v / \omega_{p}$, and with (14) for $\beta \ll 1$. Of course, with appropriate rescaling, these results apply also to the triangular JJA.
Finally, the above results for the motion of a single vortex raise the interesting question of how the coupling to spin waves, i.e., the dissipation, will be modified when increasing the vortex density. In particular, the case 
$\Phi / \Phi_{0}=\frac{1}{2}$, where $\Phi$ is the flux per plaquette and $\Phi_{0}$ the flux quantum, was studied in detail in Ref. 14 in the limit of large ohmic losses; and it was shown that for special directions of the external current, the equations of motion for the order-parameter phases can be reduced to a single one of the form known to describe a single junction, with renormalized parameters. These arguments also apply in the presence of a finite nearest-neighbor capacitance. Hence, for vanishing ohmic losses, and for a certain range of the external current, $I_{c}<I_{x}<I_{J}$, where $I_{c}$ depends on the lattice type and the current direction, there is a solution describing a vortex lattice moving without dissipation (provided $\eta \rightarrow 0$ ). It seems that the rigidity of the lattice and the "commensurability" of the external current direction are essential. At present, it is not clear how to connect this result with the above considerations, which apply in the low density limit, and to which extent the model in the continuum limit can be helpful in answering this question.

In conclusion, we remark that the present classical analysis is expected to be applicable as long as $E_{C} \ll E_{J}$, where the vortex picture makes sense, and for energies above the potential maximum. For $E_{C}<E_{J}$ but small energies, the analysis of (quantum) vortex dynamics can be based on the Hamiltonian corresponding to (15), or more sophisticated approaches, ${ }^{15}$ which result in narrow bands corresponding to a band mass much larger than the bare mass $M$. Finally, increasing the charging energy to the value $E_{C} \sim E_{J}$, a transition to an insulating charge dipole phase ${ }^{15}$ is expected; at present, it is not clear how the above picture of a vortex, emitting spin waves in its motion, is modified close to (or even beyond) the transition.

\section{ACKNOWLEDGMENTS}

We thank A. van Otterloo and U. Geigenmüller for comments, and the authors of Ref. 8 for communicating the results of their work to us. E.B.S. gratefully acknowledges the hospitality of the KfK during a recent visit, which was made possible through the DAAD program "Superconductivity."
${ }^{1}$ H. S. J. van der Zant, F. C. Fritschy, T. P. Orlando, and J. E. Mooij, Phys. Rev. Lett. 66, 2531 (1991).

${ }^{2}$ H. S. J. van der Zant, F. C. Fritschy, T. P. Orlando, and J. E. Mooij, Europhys. Lett. 18, 343 (1992).

${ }^{3}$ A. I. Larkin, Yu. N. Ovchinnikov, and A. Schmid, Physica B 152, 266 (1988).

${ }^{4}$ U. Eckern and A. Schmid, Phys. Rev. B 39, 6441 (1989).

${ }^{5} \mathrm{U}$. Eckern, in Application of Statistical and Field Theory Methods to Condensed Matter, Vol. 218 of NATO Advanced Studies Institute, Series B: Physics, edited by D. Baeriswyl et al. (Plenum, New York, 1990), p. 311; Fizika (Zagreb) 21, Suppl. 3, 253 (1989).

${ }^{6}$ T. P. Orlando, J. E. Mooij, and H. S. J. van der Zant, Phys. Rev. B 43, 10218 (1991).

${ }^{7}$ P. A. Bobbert, Phys. Rev. B 45, 7540 (1992).

${ }^{8}$ U. Geigenmüller, C. J. Lobb, and C. B. Whan, this issue, Phys. Rev. B 47, 348 (1992).
${ }^{9}$ C. J. Lobb, D. W. Abraham, and M. Tinkham, Phys. Rev. B 27, 150 (1983).

${ }^{10}$ U. Kramer, Diplom thesis, University of Karlsruhe, 1991 (unpublished).

${ }^{11}$ U. Eckern, G. Schön, and V. Ambegaokar, Phys. Rev. B 30, 6419 (1984).

${ }^{12}$ E. Simanek, Solid State Commun. 48, 1023 (1983).

${ }^{13}$ M. Oefelein, Diplom thesis, University of Karlsruhe, 1990 (unpublished).

${ }^{14}$ L. L. Sohn, M. S. Rzchowski, J. U. Free, M. Tinkham, and C. J. Lobb, Phys. Rev. B 45, 3003 (1992), and references therein.

${ }^{15}$ R. Fazio, U. Geigenmüller, and G. Schön, in Quantum Fluctuations in Mesoscopic and Macroscopic Systems, edited by $\mathbf{H}$. A. Cerdeira et al. (World Scientific, Singapore, 1991), p. 214; R. Fazio, A. van Otterloo, G. Schön, H. S. J. van der Zant, and J. E. Mooij, Helv. Physica Acta 65, 228 (1992). 